CARNETS DE Carnets de géographes

GÉOGRAPHES.

9 | 2016

Géographies des émotions

\title{
Cartographie et participation
}

Vers une pluralisation des sources de connaissance. Application à la trame verte et bleue dans le bocage bressuirais

\section{Aurélie Bousquet}

\section{(2) OpenEdition}

Journals

Édition électronique

URL : http://journals.openedition.org/cdg/625

DOI : $10.4000 /$ cdg. 625

ISSN : 2107-7266

Éditeur

UMR 245 - CESSMA

Référence électronique

Aurélie Bousquet, "Cartographie et participation », Carnets de géographes [En ligne], 9 | 2016, mis en ligne le 30 novembre 2016, consulté le 21 décembre 2020. URL : http://journals.openedition.org/cdg/ 625 ; DOI : https://doi.org/10.4000/cdg.625

\section{(c) $\Theta \Theta \Theta$}

La revue Carnets de géographes est mise à disposition selon les termes de la Licence Creative Commons Attribution - Pas d'Utilisation Commerciale - Pas de Modification 4.0 International. 


\title{
Cartographie et participation
}

\section{Vers une pluralisation des sources de connaissance. Application à la trame verte et bleue dans le bocage bressuirais}

\begin{abstract}
Aurélie Bousquet
En 2007, lors du Grenelle de l'environnement, la France décide de mettre en place la Trame Verte et Bleue (TVB) : un nouveau type de politique environnementale qui se fonde sur une approche réticulaire, et non aréale, pour endiguer «l'érosion de la biodiversité ». Le déploiement de la TVB passe par l'identification des continuités écologiques, constituées de réservoirs de biodiversité et de corridors écologiques, aux différentes échelles. Le cadre réglementaire de la TVB s'applique à trois niveaux : celui des orientations nationales; celui des Schémas Régionaux de Cohérence Écologique (SRCE); et enfin celui des communes et des intercommunalités au travers des documents d'urbanismes (SCoT, PLU, PLUi, etc).

En effet depuis la promulgation de la loi relative à la protection de la nature en 1976, les enjeux liés à la préservation de la biodiversité sont progressivement traduits sous forme de carte. Ainsi, la réalisation des TVB passe essentiellement par une identification des continuités écologiques au moyen de bases de données géographiques. L'intégration de ces données se fait dans des Systèmes d'Information Géographique en vue de la production de cartes selon des délais et des coûts acceptables. Pourtant les textes de loi et les guides méthodologiques destinés à la réalisation des TVB reprennent les principes mis en avant lors du Grenelle de l'environnement en encourageant l'essor des démarches participatives et l'implication des acteurs. Faute de méthode et de culture de la participation, le cadre réglementaire demeure peu précis sur les modalités de mise en œuvre de la participation dans la construction des TVB, alors que les attendus cartographiques sont précisément définis. Nous assistons alors à un renforcement de la sphère technique qui répond facilement aux attendus réglementaires en produisant des cartes et cela au détriment de la participation. Même en présence de dispositifs participatifs, les résultats produits par la sphère technique restent déterminants dans la construction des TVB.

Nous assistons alors à une standardisation de la production des TVB qui privilégie une forme de savoir (technique) et de représentation (cartographique) qui ne sont ni partagés ni partageables entre les individus. La question qui se pose alors est de savoir comment pluraliser les sources de connaissance et comment les intégrer aux procédures de mises en carte des continuités écologiques.

La mise en place des TVB permet d'interroger le couple participation/cartographie et nous proposons dans cette étude un cheminement méthodologique qui permet d'articuler ces deux termes a priori inconciliables. Le terrain d'étude retenu pour mener à bien cette recherche est le bocage bressuirais (Deux-Sèvres). Le Poitou-Charentes offre un contexte propice à la participation puisque depuis 2004, le Conseil Régional multiplie les démarches participatives
\end{abstract}


ce qui constitue a priori un terreau favorable pour appliquer les préconisations du Grenelle de l'environnement. De plus la région est traversée par une continuité écologique bocagère d'importance nationale. Les milieux bocagers, par leurs spécificités structurales, permettent de conduire une analyse critique des méthodes, des outils et des données mobilisés pour identifier les continuités écologiques.

Cette recherche se positionne volontairement dans une démarche inductive, exploratoire et itérative. Pour ce faire nous avons mobilisé la théorie ancrée comme approche générale. La théorie ancrée propose une manière particulière pour définir son objet de recherche, puisque celui-ci s'affine par itérations successives. La théorie ancrée alterne les phases de collecte de données et d'analyses et les ajustements entre chacune de ces phases permettent d'appréhender les objets de recherche dans une démarche souple et d'être réactif par rapport au terrain. La collecte des données a donné lieu à la mise en place et à la combinaison de plusieurs outils et méthodes permettant de développer des moyens pour intégrer les savoirs issus de la participation : entretiens semi-directifs, analyses statistiques, entretiens de cadragerecadrage, enquête photographique et ateliers de conception participative. Cet itinéraire méthodologique se compose de trois temps et est résumé dans la figure 1.

Figure 1. Itinéraire méthodologique

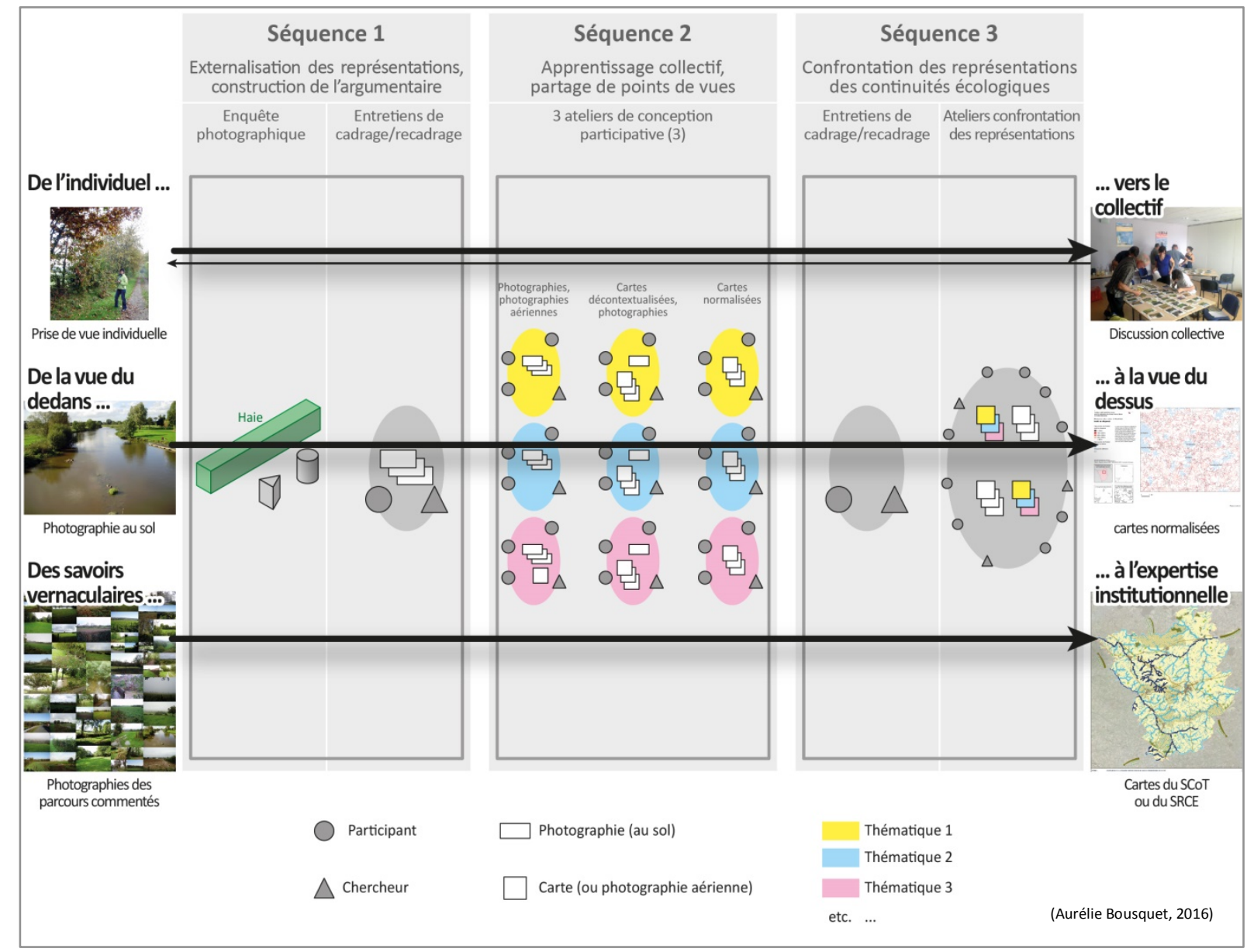

Source : Auteur, 2016

L'objectif est de produire une série de cartes selon les représentations multiples des participants qui émergent tout au long du processus. Ces trois séquences viennent répondre à cet objectif. Dans un premier temps, il s'agit d'externaliser les représentations paysagères des 
acteurs au moyen d'une enquête photographique, tout en leur permettant de construire individuellement leur argumentaire. Cette phase est essentielle pour préparer individuellement les acteurs aux débats collectifs qui vont suivre dans la séquence 2 . Le deuxième temps comprend trois ateliers de conception participative, au cours desquels s'opère la bascule de la vue tangentielle à la vue zénithale, pour cela plusieurs types de supports sont produits et mobilisés: photographies prises par les acteurs, photographies aérienne, cartes décontextualisées et cartes normalisées. Enfin un dernier temps collectif permet de confronter les cartes produites en atelier à celles produites par les institutions publiques.

L'itinéraire méthodologique mis en œuvre a conduit non pas à la production d'une carte de synthèse des continuités écologiques, mais à la production de représentations cartographiques variées qui intègre les connaissances des participants et qui renouvelle les représentations du bocage qui souvent se limitent à celle de la densité des haies. De manière plus générale, ce travail de recherche encourage à multiplier et à diversifier les expériences sur les territoires pour éviter un appauvrissement des représentations mentales et graphiques dû à une standardisation des méthodes et des outils mobilisés.

\author{
Lien électronique si la thèse est disponible en ligne \\ Discipline \\ Géographie \\ Directeur \\ Laurent Couderchet \\ Université \\ Université Bordeaux Montaigne \\ Membres du jury de thèse, soutenue le 11 avril 2016
}

- Laurent Couderchet, Professeur des Universités en géographie, Université Bordeaux Montaigne (Directeur)

- Corinne Larrue, Professeure des Universités en aménagement de l'espace et urbanisme, École d'urbanisme de Paris (Rapporteuse)

- Sylvie Lardon, Directrice de recherche INRA, Professeure AgroParisTech (Rapporteuse)

- Thierry Joliveau, Professeur des Universités en géographie, Université Jean Monnet de Saint-Étienne

(Président du jury)

- Béatrice Collignon, Professeure des Universités en géographie, Université Bordeaux Montaigne (Examinatrice)

- Jean-Pierre Thibault, Inspecteur général de l'environnement et du développement durable, Ministère de

l'Écologie du Développement durable et de l'Énergie (Examinateur)

Situation professionnelle actuelle

ATER en géographie, Université Bordeaux Montaigne

Courriel de l'auteur

aurelie.bousquet33[at]hotmail.fr 